\title{
Oxytocin in survivors of childhood-onset craniopharyngioma
}

\section{Anna M. M. Daubenbüchel, Anika}

Hoffmann, Maria Eveslage, Jale Özyurt, Kristin Lohle, Julia Reichel, Christiane M. Thiel, et al.

\section{Endocrine}

International Journal of Basic and Clinical Endocrinology

ISSN 1355-008X

\section{Volume 54}

Number 2

Endocrine (2016) 54:524-531

DOI 10.1007/s12020-016-1084-5
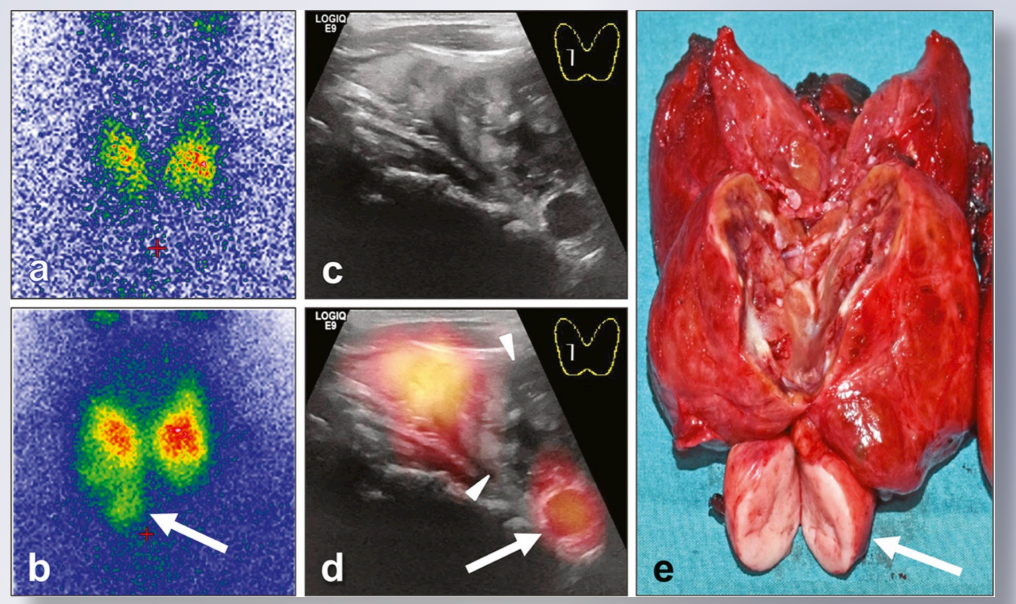
Your article is protected by copyright and all rights are held exclusively by Springer Science +Business Media New York. This e-offprint is for personal use only and shall not be selfarchived in electronic repositories. If you wish to self-archive your article, please use the accepted manuscript version for posting on your own website. You may further deposit the accepted manuscript version in any repository, provided it is only made publicly available 12 months after official publication or later and provided acknowledgement is given to the original source of publication and a link is inserted to the published article on Springer's website. The link must be accompanied by the following text: "The final publication is available at link.springer.com". 


\title{
Oxytocin in survivors of childhood-onset craniopharyngioma
}

\author{
Anna M. M. Daubenbüchel ${ }^{1,2} \cdot$ Anika Hoffmann $^{1} \cdot$ Maria Eveslage $^{3} \cdot$ Jale Özyurt $^{4}$. \\ Kristin Lohle $^{1} \cdot$ Julia Reichel $^{1} \cdot$ Christiane M. Thiel $^{4,5} \cdot$ Henri Martens $^{6}$. \\ Vincent Geenen $^{6} \cdot$ Hermann L. Müller ${ }^{1}$
}

Received: 19 July 2016 / Accepted: 9 August 2016 / Published online: 1 September 2016

(C) Springer Science+Business Media New York 2016

\begin{abstract}
Quality of survival of childhood-onset craniopharyngioma patients is frequently impaired by hypothalamic involvement or surgical lesions sequelae such as obesity and neuropsychological deficits. Oxytocin, a peptide hormone produced in the hypothalamus and secreted by posterior pituitary gland, plays a major role in regulation of behavior and body composition. In a crosssectional study, oxytocin saliva concentrations were analyzed in 34 long-term craniopharyngioma survivors with and without hypothalamic involvement or treatmentrelated damage, recruited in the German Childhood Craniopharyngioma Registry, and in 73 healthy controls, attending the Craniopharyngioma Support Group Meeting 2014. Oxytocin was measured in saliva of craniopharyngioma patients and controls before and after standardized breakfast and associations with gender, body mass index, hypothalamic involvement, diabetes insipidus, and
\end{abstract}

Hermann L. Müller

mueller.hermann@klinikum-oldenburg.de

1 Department of Pediatrics, Klinikum Oldenburg AöR, Medical Campus University Oldenburg, 26133 Oldenburg, Germany

2 University Medical Centre Groningen (UMCG), University of Groningen, 9712 Groningen, The Netherlands

3 Institute of Biostatistics and Clinical Research, University of Münster, 48149 Münster, Germany

4 Biological Psychology Lab, Department of Psychology, Faculty of Medicine and Health Sciences, Carl von Ossietzky University, 26129 Oldenburg, Germany

5 Research Center Neurosensory Science and Cluster of Excellence "Hearing4all", Carl von Ossietzky University, 26129 Oldenburg, Germany

6 GIGA-I3 Center of Immunoendocrinology, University of Liege Liege-Sart Tilman, 4000 Liege, Belgium irradiation were analyzed. Patients with preoperative hypothalamic involvement showed similar oxytocin levels compared to patients without hypothalamic involvement and controls. However, patients with surgical hypothalamic lesions grade 1 (anterior hypothalamic area) presented with lower levels $(p=0.017)$ of oxytocin under fasting condition compared to patients with surgical lesion of posterior hypothalamic areas (grade 2) and patients without hypothalamic lesions (grade 0). Craniopharyngioma patients' changes in oxytocin levels before and after breakfast correlated $(p=0.02)$ with their body mass index. Craniopharyngioma patients continue to secrete oxytocin, especially when anterior hypothalamic areas are not involved or damaged, but oxytocin shows less variation due to nutrition. Oxytocin supplementation should be explored as a therapeutic option in craniopharyngioma patients with hypothalamic obesity and/or behavioral pathologies due to lesions of specific anterior hypothalamic areas. Clinical trial number: KRANIOPHARYNGEOM 2000/2007(NCT00258453; NCT01272622).

Keywords Craniopharyngioma $\cdot$ Hypothalamus $\cdot$ Obesity Oxytocin · Behavior

\section{Introduction}

Childhood-onset craniopharyngioma (CP) are rare intracranial embryonal malformations of the sellar region arising from remnants of Rathke's pouch [1]. CP show low-grade histological malignancy (WHO ${ }^{\circ}$ I) and frequently effect hypothalamic and pituitary regions due to their location [2]. This hypothalamic involvement (HI) of $\mathrm{CP}$ and/or 
treatment-related lesions of this region usually result in endocrine deficiencies, neuropsychological deficits, pathological patterns of eating behavior, and obesity with major negative impact on prognosis and quality of life in surviving patients [3-7]. Substitution therapy for endocrine deficiencies-hydrocortisone, sex steroids, growth hormone, vasopressin, and thyroid hormones-is an elemental part in CP treatment for patients with disturbances involving the hypothalamic and pituitary regions [2].

The peptide hormone oxytocin, which is primarily synthesized in the paraventricular and supraoptic nuclei of the hypothalamus and released by the posterior pituitary, has not been analyzed in the context of CP before this study [8]. It can be assumed that oxytocin synthesis and release is disturbed in patients with $\mathrm{CP}$, similar to the other hormones of the pituitary gland. Oxytocin is similar to the peptide hormone vasopressin, as its structure differs only by two amino acids [9]. However, in contrast to vasopressin, whose function is well understood and the clinical symptoms in vasopressin-deficient patients are well known [10], little is known about the role of oxytocin in CP patients. Oxytocin is involved in metabolism [11] and in the regulation of a wide variety of social and non-social behaviors such as nonsocial memory, anxiety, depression, and stress [12-16].

To evaluate the role of oxytocin in $\mathrm{CP}$ patients, we conducted a pilot cross-sectional study analyzing a small group of patients recruited in the German Craniopharyngioma Registry to assess oxytocin concentrations in saliva of patients with childhood-onset $\mathrm{CP}$ in comparison to healthy controls.

\section{Patients and methods}

\section{Patients}

This study analyzed patients with adamantinomatous childhood-onset $\mathrm{CP}$, recruited in the German Craniopharyngioma Registry and prospectively evaluated in the multicenter trials HIT Endo and KRANIOPHARYNGEOM 2000/ 2007 (Clinical Trial No.: NCT00258453; NCT01272622), who attended the 17th Annual Meeting of the German Craniopharyngioma Support Group, Bad Sassendorf, Germany, 2014. Thirty-four of 40 patients $(85 \%)$ attending this meeting agreed to participate in the study. These $34 \mathrm{CP}$ patients (19 female, 15 male) had been initially diagnosed at a median age of 10.5 years (range: $0.02-18.0$ years). The study cohort $(n=34)$ showed similar characteristics in terms of age and gender distribution, degree of surgical resection, HI, and hypothalamic surgical lesions when compared with the total cohort of $\mathrm{CP}$ patients recruited in the German Craniopharyngioma Registry.
Oxytocin concentrations were analyzed at a median patient age of 20 years (range $7-41$ years), with a median interval between initial CP diagnosis and this study of 11.0 years (range: 0.5-31.0 years). They were analyzed for height, weight, and body mass index (BMI), as well as oxytocin concentrations in saliva, for which samples were taken shortly before and $60 \mathrm{~min}$ after breakfast. For the oxytocin concentration comparisons, a standardized and comparable breakfast for all participants was ensured (approx. 10-15 kcal/kg body weight; 8 a.m.). The study was conducted during the 17thAnnual Meeting of the German Craniopharyngioma Support Group, Bad Sassendorf, Germany, 2014. A total of 73 healthy children, adolescents, and adults, also attending the 17th Annual Craniopharyngioma Support Group Meeting, 2014, served as controls.

Body composition and the degree of obesity were evaluated by calculating the BMI standard deviation score (SDS) according to the references of Rolland-Cachera et al. [17]. The histological diagnosis of anadamantinomatous CP was confirmed by reference assessment in all cases. HI of $\mathrm{CP}$ was assessed by magnetic resonance imaging (MRI), computed tomography, and/or microscopic inspection during surgery. Preoperative HI was defined as involvement of hypothalamic structures either by tumor growth into the hypothalamus or displacement of hypothalamic structures by the tumor. Surgical hypothalamic lesions were assessed on postoperative MRI and reference-confirmed by a neuroradiologist blinded for the clinical data based on a previously published grading system [18, 19].

The study was approved by the local standing committee on ethical practice and written parental and/or patient consent was obtained in all cases.

\section{Oxytocin analysis in saliva}

We compared saliva levels of the $34 \mathrm{CP}$ patients with the saliva of a healthy control group consisting of 41 female and 32 male participants with a median age of 39 years (range 8-63 years). Oxytocin saliva levels were measured under fasting conditions as well as directly after a standardized breakfast meal. In addition, we quantified changes in oxytocin levels before and after breakfast ( $\Delta$ oxytocin is defined as the difference in oxytocin after breakfast-oxytocin before breakfast). To measure oxytocin saliva levels, saliva was collected from each participant (patients and controls) before and after breakfast using a standardized method for saliva sampling. A white cylindrical swab was chewed gently for $15 \mathrm{~min}$ by each participant and then returned with the absorbed saliva into a salivette-a saliva collection device. All salivettes were placed immediately on ice and kept cool till centrifugation. After centrifugation, patient saliva samples were stored frozen until analysis. Oxytocin concentrations in saliva were measured by 
immunoassay [20]. Briefly, a competition between biotinylated oxytocin used as tracer and synthetic oxytocin provides a displacement curve after incubation with streptavidin-horseradish peroxidase and revelation with TMB 3,3',5,5'-Tetramethylbenzidine. Filtration on Amicon Ultracel-2, membrane of $3 \mathrm{kDa}$ (cutoff 3000)-both from Merck Millipore-was performed in order to reduce interferences. Filtration recovery was measured as $68.4 \pm 14.4 \%$ (coefficient of variance: $21 \%$ ) of $10 \mathrm{pg}$ added to hormonefree plasma. Combined intra-assays standard deviations (SD) for the enzyme immunoassay (EIA) $(n=10$ in duplicates and triplicates) were $1.05 \%$ at $7.2 \mathrm{pg} / \mathrm{ml}$, $1.94 \%$ at $20 \mathrm{pg} / \mathrm{ml}$, and $1.58 \%$ at $200 \mathrm{pg} / \mathrm{ml}$. Detection limit ( \pm 2 SD from zero) was typically $0.5 \mathrm{pg} / \mathrm{ml}$ and always under $1 \mathrm{pg} / \mathrm{ml}$.

\section{Statistical analyses}

Statistical analysis was performed with SPSS $23^{\circledR}$ for Windows (IBM Corporation, Somers, NY, USA) and R version 3.1.0. For metrical variables, the comparison of two and three independent groups was performed with the Mann-Whitney $U$ test and the Kruskal-Wallis test, respectively. Post-hoc pairwise comparisons were performed using Dunn's test. For comparison of groups concerning categorical variables, Fisher's exact test was used. Correlation was calculated with the Pearson correlation coefficient. The local significance level was set to 0.05 . Nevertheless, all inferential statistics were intended to be exploratory (hypotheses generating), not confirmatory, and were interpreted accordingly. Therefore, no adjustment for multiple testing was applied.

\section{Results}

Oxytocin levels in saliva of $34 \mathrm{CP}$ patients were analyzed. Proven initial HI was detected in 20 of the 34 cases (59\%) before first surgical treatment of CP. Complete surgical resection was achieved at initial diagnosis in 10 of $28 \mathrm{CP}$ patients (36\%), resection data being available for only these 28 patients. A total 14 of the $34 \mathrm{CP}$ patients $(41 \%)$ received irradiation. Based on desmopressin medication, 28 of $34 \mathrm{CP}$ patients $(82 \%)$ had presented with diabetes insipidus by the time this study was initiated. Gender related differences in terms of HI, degree of resection, irradiation and diabetes insipidus were not detectable.

We compared oxytocin saliva levels in CP patients with concentrations in saliva of healthy controls. Oxytocin saliva levels were measured under fasting conditions as well as directly after a standardized breakfast meal. In addition, we quantified changes in oxytocin levels before and after breakfast ( $\Delta$ oxytocin). No significant differences in the

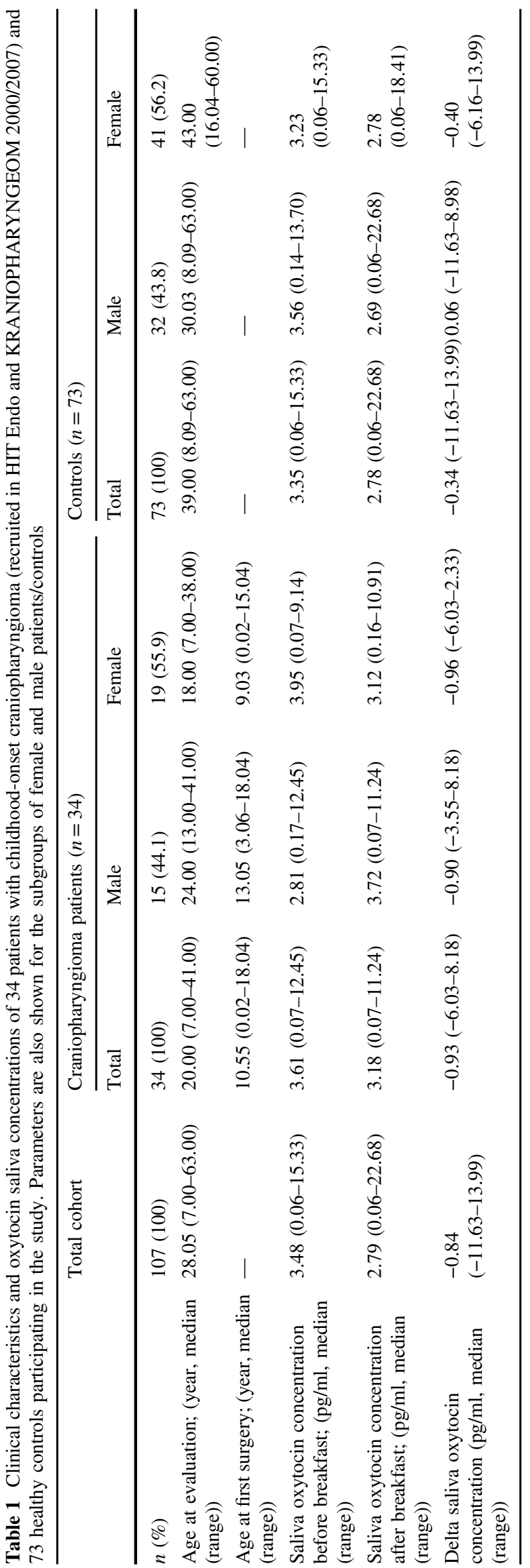


oxytocin levels were detected in the CP patients compared to the controls, indicating that $\mathrm{CP}$ patients were still able to produce and secrete the hormone oxytocin (Table 1). In addition, the subgroup of 20 patients with proven initial HI did not show any significant differences compared to patients without initial $\mathrm{HI}$ and the controls (Fig. 1a-c).

Grading of the post-surgical hypothalamic lesion was possible in 27 of the 34 patients $(79 \%)$. This analysis showed seven patients had no treatment-related hypothalamic lesion; six patients showed a surgical hypothalamic lesion grade 1 , which is defined as a damage of the anterior parts of the hypothalamic region, leaving the corpora mammillaria intact. The grading analysis further revealed 14 patients with hypothalamic lesions grade 2, which is defined as a damage of the anterior and posterior parts of the hypothalamic region. By comparison of these subgroups, patients with hypothalamic lesion grade 1 showed a significant lower level of oxytocin before breakfast, compared to patients without hypothalamic lesion and compared to patients with hypothalamic lesion grade 2 of the anterior and posterior hypothalamus $(p=0.017)$. Patients with hypothalamic lesion grade 2 did not differ in terms of oxytocin levels before breakfast compared to patients without hypothalamic lesion. In the oxytocin measurements after breakfast, again the patients with hypothalamic lesion grade 1 showed a lower oxytocin level compared to hypothalamic lesion grade 2 patients, but without reaching a significant level. In the analysis of $\Delta$ oxytocin before and after breakfast, no differences were detectable (Fig. 2a-c).

By comparison of changes in oxytocin saliva levels before and after breakfast ( $\Delta$ oxytocin), no significant differences could be measured in patients having undergone radiotherapy (Fig. 3a) or in those with diabetes insipidus (Fig. 3b). Due to the important role of oxytocin in females, we compared oxytocin expression in male and female CP patients and controls. Again, no significant differences could be detected (Fig. 3c).

However, in our analysis of further clinical parameters, it turned out that changes in oxytocin levels ( $\Delta$ oxytocin) before and after breakfast correlated significantly $(p=0.02)$ with BMI in CP patients. CP patients with a high BMI showed smaller oxytocin changes. In controls, no significant correlation between BMI SDS and $\Delta$ oxytocin (before/after breakfast) could be detected (Fig. 4).

\section{Discussion}

The present study reports the first analysis of oxytocin saliva concentrations in adamantinomatous childhood-onset CP patients. This is significant, as oxytocin synthesis takes place in the hypothalamus and is released mainly via the pituitary gland, and these structures are often damaged by
$\mathrm{CP}$ either from tumor involvement or its surgical treatment. Therefore, it was suspected that oxytocin synthesis and release would be disturbed in CP patients. However, we could not detect a lack of oxytocin in $\mathrm{CP}$ patients. In addition, we detected no difference in oxytocin saliva concentrations compared to the healthy control group. The conservation of oxytocin synthesis in CP patients might be explained by a residual or preserved function of hypothalamic structures and/or a taking over of synthesis function by other neuronal regions. It has been described that-in addition to the synthesis in the paraventricular and supraoptic nuclei of the hypothalamus-lesser amounts of oxytocin are generated in the bed nucleus of the striaterminalis, medial preoptic area, and lateral amygdala, depending on the species [21]. The release of oxytocin in humans is known not to happen exclusively via axonal secretion in the posterior pituitary but also from the dendrites (opposed to the axons) for intracerebral release [2224]. By discrete grading and comparisons of $\mathrm{CP}$ patients with grade 1 hypothalamic surgical damage (damage exclusively to the anterior hypothalamic structures) and grade 2 (damage of anterior and posterior hypothalamic regions), we found that patients with grade 1 damage only to the anterior hypothalamus had a lower oxytocin level before breakfast compared to patients with grade 2 damage and to patients without any hypothalamic damage. This finding is, however, inconclusive, because only six patients were categorized with grade 1 hypothalamic damage. Nevertheless, our comparison of results still lead us to speculate that in some patients, oxytocin production and release is diminished by hypothalamic damage due to surgical treatment. The localization of the paraventricular and supraoptic nuclei of the hypothalamus might be the reason for preservation of oxytocin production since both nuclei are localized in the anterior superior part of the hypothalamus. Growth of $\mathrm{CP}$, however, typically takes place from an anterior inferior to an upwards posterior direction. Thus, in patients with $\mathrm{HI}$, the anterior inferior (tuberal region) of the hypothalamus is attacked first [25]. Depending on the direction of growth and tumor size, the paraventricular and supraoptic nuclei may frequently be spared, resulting in preservation of oxytocin synthesis.

In our small group of $34 \mathrm{CP}$ patients, we were indeed able to detect a negative correlation between BMI and changes in oxytocin levels before and after breakfast. This finding links oxytocin to the main problem of $\mathrm{CP}$ survivors with $\mathrm{HI}$, which is severe hypothalamic obesity and its consequent long-term negative impact on quality of life [1]. It is already well established that the hypothalamus is the regulatory center for feeding and satiety [26]. Oxytocin has also been linked to energy homeostasis mechanisms in animals, where it acts as a strong inhibitor of food intake and affects energy expenditure and glucose homeostasis [27-31]. Oxytocin 
a)

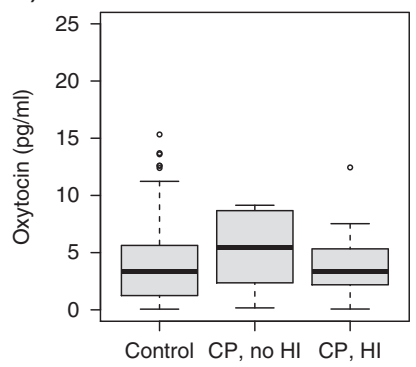

b)

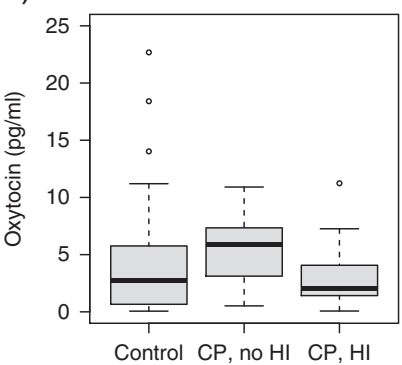

c)

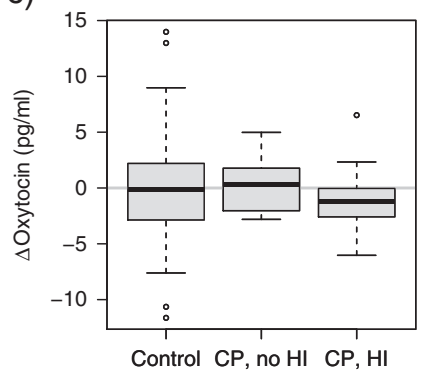

Fig. 1 Oxytocin saliva concentrations $(\mathrm{pg} / \mathrm{ml})$ in childhood-onset craniopharyngioma (CP) patients recruited in HIT Endo and KRANIOPHARYNGEOM 2000/2007 before breakfast (a) and 60 min after standardized breakfast (b) and $\Delta$ oxytocin saliva concentrations (oxytocin concentration after standardized breakfast vs. oxytocin concentration before breakfast) (c) in CP patients with and without preoperative hypothalamic involvement (HI) and healthy controls. The horizontal line in the middle of the box depicts the median. The top and bottom edges of the box, respectively, mark the 25 th and 75 th percentiles. Whiskers indicate the range of values that fall within 1.5 box lengths

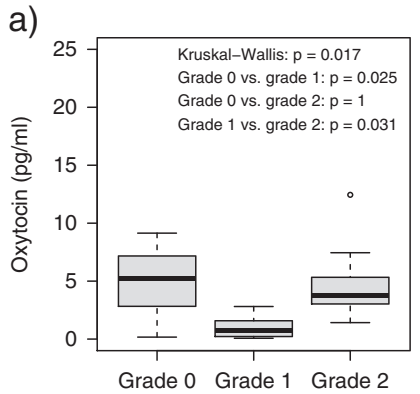

b)

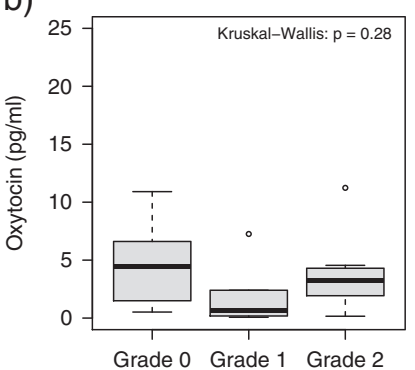

c)

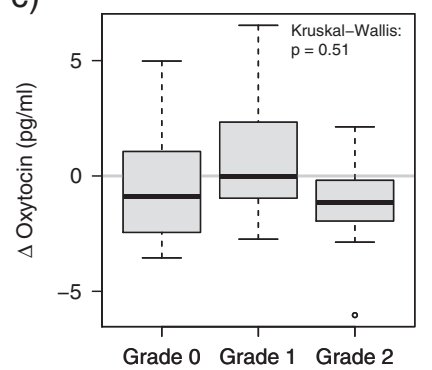

Fig. 2 Oxytocin saliva concentrations (pg/ml) in childhood-onset craniopharyngioma (CP) patients recruited in HIT Endo and KRANIOPHARYNGEOM 2000/2007 before breakfast (a) and 60 min after standardized breakfast (b) and $\Delta$ oxytocin saliva concentrations (oxytocin concentration after standardized breakfast vs. oxytocin concentration before breakfast) (c) in CP patients with different grades (grade 0-2) of surgical hypothalamic lesions according to the grading system of Müller et al. [18, 19]. Grade 0 no hypothalamic lesions; grade 1 surgical lesions involving anterior hypothalamic areas, sparing the mammillary bodies; grade 2 surgical lesions involving anterior and posterior hypothalamic areas involving the mammillary bodies. The horizontal line in the middle of the box depicts the median. The top and bottom edges of the box, respectively, mark the 25 th and 75 th percentiles. Whiskers indicate the range of values that fall within 1.5 box lengths a)

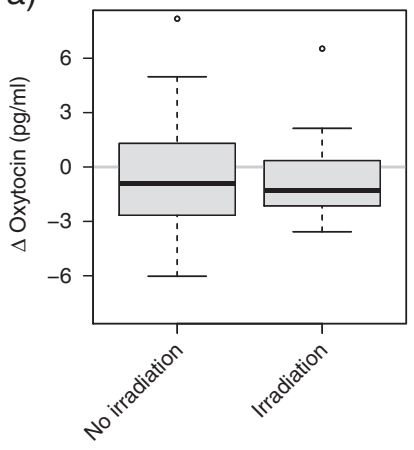

b)

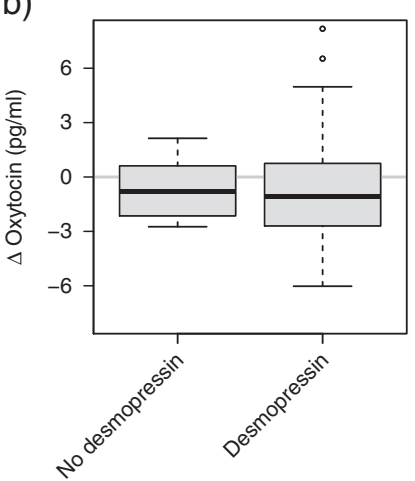

c)

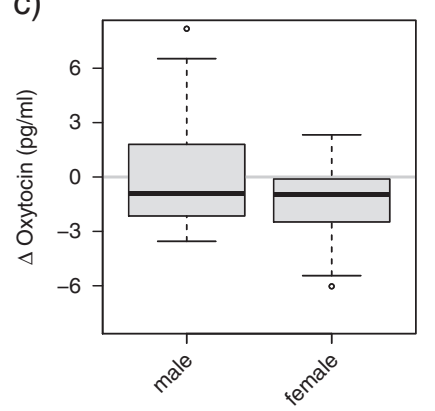

Fig. 3 Expression of $\Delta$ oxytocin saliva concentrations (oxytocin concentration after standardized breakfast vs. oxytocin concentration before breakfast; $\mathrm{pg} / \mathrm{ml}$ ) in craniopharyngioma patients recruited in HIT Endo and KRANIOPHARYNGEOM 2000/2007 with and without irradiation therapy (a) with and without diabetes insipidus (b) and

has also been shown to play an important role in metabolism and energy balance in humans [32, 33], such as reducing caloric intake with a preferential effect on fat regarding gender (c). The horizontal line in the middle of the box depicts the median. The top and bottom edges of the box, respectively, mark the 25th and 75th percentiles. Whiskers indicate the range of values that fall within 1.5 box-lengths

intake [11] and reward-driven food intake in humans [34]. These findings are in line with our finding that reduced levels of delta oxytocin correlate with a high BMI in $\mathrm{CP}$ 


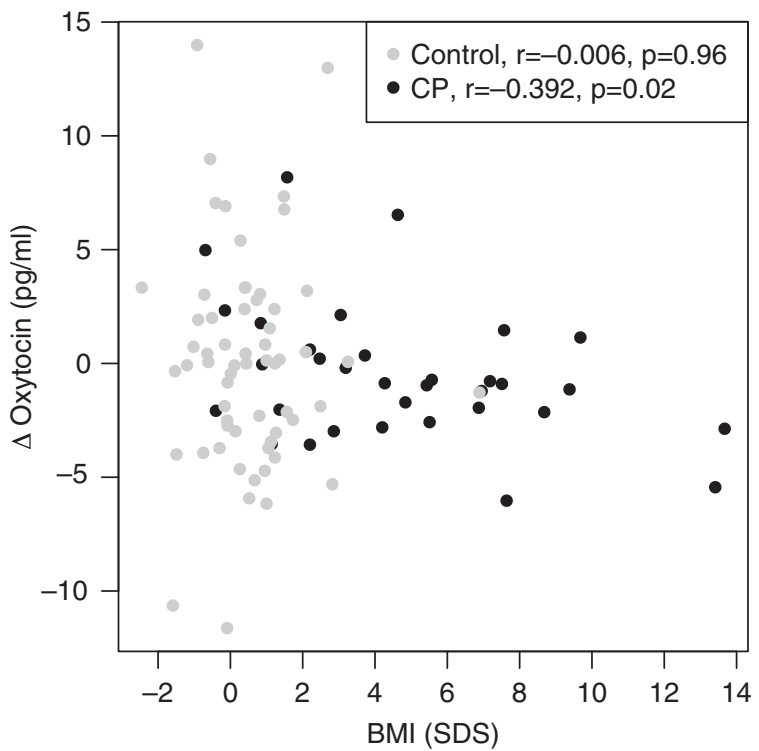

Fig. 4 Correlation of $\Delta$ oxytocin saliva concentrations (oxytocin concentration $60 \mathrm{~min}$ after standardized breakfast vs. oxytocin concentration before breakfast; $\mathrm{pg} / \mathrm{ml}$ ) with body mass index (BMI) SDS in childhood-onset craniopharyngioma patients (black dots) recruited in HIT Endo and KRANIOPHARYNGEOM 2000/2007 and controls (grey dots). $r=$ Pearson correlation coefficient

patients. We could not demonstrate this delta oxytocin/BMI correlation in the healthy control group, which might be explained by the lower variability of BMI. In a recent study with newly diagnosed type 2 diabetic patients, serum oxytocin levels were lower compared to the control group with normal glucose tolerance. Serum oxytocin levels were negatively correlated with BMI, waist circumference, and other parameters of pathological insulin metabolism [35]. This correlation of low oxytocin levels and a high BMI in diabetes type 2 patients [35], or minor changes of oxytocin saliva concentrations in reaction to nutrition (in this study, a standardized breakfast) and a high BMI, might indicate that oxytocin is a potential pharmaceutical agent for obesity treatment. Indeed, this has been tried in patients with Prader-Willi syndrome (PWS), a genetic disease with hypothalamic dysfunction, severe obesity, and hyperphagia as well as cognitive and behavioral problems. Oxytocin supplementation has been tested in a randomized placebocontrolled trial in 24 PWS patients [16]. After a single intranasal administration of oxytocin, improved patients' trust in others as well as decreased tendencies towards depressed moods could be observed. Not surprisingly, no effects on obesity could be detected from this single oxytocin administration [16]. This study demonstrates that oxytocin therapy might be an option for diseases with hypothalamic dysfunction-induced behavioral disturbances and obesity. This therapeutic potential is further reinforced by a recent case study about a parent having observed neurobehavioral and pro-social improvement with oxytocin therapy following surgical resection of their child's CP [36], supporting earlier reports on oxytocin effects on social cognition and affiliative behaviour [13-15].

There is a significant amount of extant literature pertaining to the role that oxytocin plays in brain function, and that one of the major mechanisms associated with oxytocin is inhibition of the amygdala, reducing feelings of fear and anxiety [37]. Other studies have found that intranasal oxytocin may activate reward pathways in the globus pallidus, the striatum, middle frontal gyrus, medial prefrontal cortex, right orbitofrontal cortex, and the left superior temporal sulcus in autistic children [38]. However, in order to recommend intranasal application of oxytocin as a general therapeutic option for CP-induced hypothalamic dysfunction, further research is needed regarding the role oxytocin plays in human metabolism and behavioural functionality.

Limitations of our study are related to the small size of our cohort and difficulties of oxytocin measurement. First, it remains a matter of discussion whether peripherally measured oxytocin is indicative for central release patterns and activities within the brain [39]. Some studies suggest that certain stimuli lead to coordinated release of central and peripheral oxytocin, whereas other studies indicate that certain stimuli change central levels while leaving peripheral levels unchanged [40]. In addition, the reliability of oxytocin measurement is still under debate [39], and issues have been raised regarding the importance of preanalytical procedures [41]. In this study, we used saliva samples measured with EIA after extraction. To avoid analytical inconsistencies, we measured healthy volunteers as controls, also participating at the 17th Annual Craniopharyngioma Meeting, 2014, under the same nutrition conditions as the $\mathrm{CP}$ patients and analyzed all saliva samples using identical laboratory procedures. We were not able to analyze other metabolic parameters such as glucose, lipid and insulin levels and their association with pre- and post-prandial oxytocin concentrations in saliva. Based on the results of this pilot study, further investigations including analyses of these parameters are part of an upcoming new study in the context of the German Craniopharyngioma Registry.

We conclude that CP patients are able to produce and secrete the hormone oxytocin, even when pituitary and hypothalamic structures are damaged. However, patients with hypothalamic damage grade 1 , which involves damage only to the anterior hypothalamic areas, present with a significant lower fasting level of oxytocin. In addition, changes in oxytocin levels ( $\Delta$ oxytocin) before and after standardized breakfast correlate significantly $(p=0.02)$ with $\mathrm{BMI}$ in $\mathrm{CP}$ patients, demonstrating that patients with hypothalamic obesity show less variation in oxytocin secretion due to nutrition. Accordingly, oxytocin 
supplementation might be a therapeutic optionin CP patients with hypothalamic obesity and/or neurobehavioral deficits due to specific hypothalamic damage in the anterior hypothalamic area.

Acknowledgements We thank all patients who participated in this study. We are grateful for the help of Margarita Neff-Heinrich, Göttingen, Germany, in proofreading and editing the manuscript. H. L. Müller is supported by the German Childhood Cancer Foundation, Bonn, Germany.

\section{Compliance with ethical standards}

Conflict of interest The authors declare that they have no conflict of interest.

\section{References}

1. H.L. Muller, Craniopharyngioma. Endocr. Rev. 35(3), 513-543 (2014). doi:10.1210/er.2013-1115

2. H.L. Muller, Consequences of craniopharyngioma surgery in children. J. Clin. Endocrinol. Metab. 96(7), 1981-1991 (2011). doi:10.1210/jc.2011-0174

3. O.M. Dekkers, N.R. Biermasz, J.W. Smit, L.E. Groot, F. Roelfsema, J.A. Romijn, A.M. Pereira, Quality of life in treated adult craniopharyngioma patients. Eur. J. Endocrinol. 154(3), 483-489 (2006). doi:10.1530/eje.1.02114

4. A.S. Sterkenburg, A. Hoffmann, U. Gebhardt, M. Warmuth-Metz, A.M. Daubenbuchel, H.L. Muller, Survival, hypothalamic obesity, and neuropsychological/psychosocial status after childhoodonset craniopharyngioma: newly reported long-term outcomes. Neuro Oncol. 17(7), 1029-1038 (2015). doi:10.1093/neuonc/ nov044

5. A. Bereket, W. Kiess, R.H. Lustig, H.L. Muller, A.P. Goldstone, R. Weiss, Y. Yavuz, Z. Hochberg, Hypothalamic obesity in children. Obes. Rev. 13(9), 780-798 (2012). doi:10.1111/j.1467789X.2012.01004.X

6. G. Zada, E.R. Laws, Surgical management of craniopharyngiomas in the pediatric population. Horm. Res. Paediatr 74(1), 62-66 (2010). doi:10.1159/000309349

7. H.L. Muller, Craniopharyngioma and hypothalamic injury: latest insights into consequent eating disorders and obesity. Curr. Opin. Endocrinol. Diabetes Obes. 23(1), 81-89 (2016). doi:10.1097/ MED.0000000000000214

8. A. Acevedo-Rodriguez, S.K. Mani, R.J. Handa, Oxytocin and estrogen receptor beta in the brain: an overview. Front. Endocrinol. (Lausanne) 6, 160 (2015). doi:10.3389/fendo.2015.00160

9. H.J. Lee, A.H. Macbeth, J.H. Pagani, W.S. Young 3rd, Oxytocin: the great facilitator of life. Prog. Neurobiol. 88(2), 127-151 (2009). doi:10.1016/j.pneurobio.2009.04.001

10. E. Frank, R. Landgraf, The vasopressin system--from antidiuresis to psychopathology. Eur. J. Pharmacol. 583(2-3), 226-242 (2008). doi:10.1016/j.ejphar.2007.11.063

11. E.A. Lawson, D.A. Marengi, R.L. DeSanti, T.M. Holmes, D.A. Schoenfeld, C.J. Tolley, Oxytocin reduces caloric intake in men. Obesity (Silver Spring) 23(5), 950-956 (2015). doi:10.1002/ oby. 21069

12. M. Olff, J.L. Frijling, L.D. Kubzansky, B. Bradley, M.A. Ellenbogen, C. Cardoso, J.A. Bartz, J.R. Yee, M. van Zuiden, The role of oxytocin in social bonding, stress regulation and mental health: an update on the moderating effects of context and interindividual differences. Psychoneuroendocrinology 38(9), 1883-1894 (2013). doi:10.1016/j.psyneuen.2013.06.019

13. H.E. Ross, L.J. Young, Oxytocin and the neural mechanisms regulating social cognition and affiliative behavior. Front. Neuroendocrinol. 30(4), 534-547 (2009). doi:10.1016/j.yfrne.2009.05.004

14. T.R. Insel, The challenge of translation in social neuroscience: a review of oxytocin, vasopressin, and affiliative behavior. Neuron 65(6), 768-779 (2010). doi:10.1016/j.neuron.2010.03.005

15. P.S. Churchland, P. Winkielman, Modulating social behavior with oxytocin: how does it work? What does it mean? Horm. Behav. 61(3), 392-399 (2012). doi:10.1016/j.yhbeh.2011.12.003

16. M. Tauber, C. Mantoulan, P. Copet, J. Jauregui, G. Demeer, G. Diene, B. Roge, V. Laurier, V. Ehlinger, C. Arnaud, C. Molinas, D. Thuilleaux, Oxytocin may be useful to increase trust in others and decrease disruptive behaviours in patients with Prader-Willi syndrome: a randomised placebo-controlled trial in 24 patients. Orphanet J. Rare Dis. 6, 47 (2011). doi:10.1186/1750-1172-6-47

17. M.F. Rolland-Cachera, T.J. Cole, M. Sempe, J. Tichet, C. Rossignol, A. Charraud, Body mass index variations: centiles from birth to 87 years. Eur. J. Clin. Nutr. 45(1), 13-21 (1991)

18. H.L. Muller, U. Gebhardt, C. Teske, A. Faldum, I. Zwiener, M. Warmuth-Metz, T. Pietsch, F. Pohl, N. Sorensen, G. Calaminus, Post-operative hypothalamic lesions and obesity in childhood craniopharyngioma: results of the multinational prospective trial KRANIOPHARYNGEOM 2000 after 3-year follow-up. Eur. J. Endocrinol. 165(1), 17-24 (2011). doi:10.1530/EJE-11-0158

19. H.L. Muller, U. Gebhardt, A. Faldum, M. Warmuth-Metz, T. Pietsch, F. Pohl, G. Calaminus, N. Sorensen, Xanthogranuloma, Rathke's cyst, and childhood craniopharyngioma: results of prospective multinational studies of children and adolescents with rare sellar malformations. J. Clin. Endocrinol. Metab. 97(11), 3935-3943 (2012). doi:10.1210/jc.2012-2069

20. C. Pequeux, J.C. Hendrick, M.T. Hagelstein, V. Geenen, J.J. Legros, Novel plasma extraction procedure and development of a specific enzyme-immunoassay of oxytocin: application to clinical and biological investigations of small cell carcinoma of the lung. Scand. J. Clin. Lab. Invest. 61(5), 407-415 (2001)

21. W.S. Young 3rd, H. Gainer, Transgenesis and the study of expression, cellular targeting and function of oxytocin, vasopressin and their receptors. Neuroendocrinology 78(4), 185-203 (2003). doi:10.1159/000073702

22. M. Ludwig, N. Sabatier, P.M. Bull, R. Landgraf, G. Dayanithi, G. Leng, Intracellular calcium stores regulate activity-dependent neuropeptide release from dendrites. Nature 418(6893), 85-89 (2002). doi:10.1038/nature00822

23. D.V. Pow, J.F. Morris, Dendrites of hypothalamic magnocellular neurons release neurohypophysial peptides by exocytosis. Neuroscience 32(2), 435-439 (1989)

24. D.A. Baribeau, E. Anagnostou, Oxytocin and vasopressin: linking pituitary neuropeptides and their receptors to social neurocircuits. Front. Neurosci 9, 335 (2015). doi:10.3389/fnins.2015.00335

25. H.G. Bauer, Endocrine and other clinical manifestations of hypothalamic disease; a survey of 60 cases, with autopsies. J. Clin. Endocrinol. Metab. 14(1), 13-31 (1954). doi:10.1210/jcem14-1-13

26. G.J. Morton, D.E. Cummings, D.G. Baskin, G.S. Barsh, M.W. Schwartz, Central nervous system control of food intake and body weight. Nature 443(7109), 289-295 (2006). doi:10.1038/ nature 05026

27. J.M. Ho, J.E. Blevins, Coming full circle: contributions of central and peripheral oxytocin actions to energy balance. Endocrinology 154(2), 589-596 (2013). doi:10.1210/en.2012-1751

28. G. Zhang, H. Bai, H. Zhang, C. Dean, Q. Wu, J. Li, S. Guariglia, Q. Meng, D. Cai, Neuropeptide exocytosis involving synaptotagmin-4 and oxytocin in hypothalamic programming of 
body weight and energy balance. Neuron 69(3), 523-535 (2011). doi:10.1016/j.neuron.2010.12.036

29. G.J. Morton, B.S. Thatcher, R.D. Reidelberger, K. Ogimoto, T. Wolden-Hanson, D.G. Baskin, M.W. Schwartz, J.E. Blevins, Peripheral oxytocin suppresses food intake and causes weight loss in diet-induced obese rats. Am. J. Physiol. Endocrinol. Metab. 302(1), E134-144 (2012). doi:10.1152/ajpendo.00296.2011

30. B.R. Olson, M.D. Drutarosky, M.S. Chow, V.J. Hruby, E.M. Stricker, J.G. Verbalis, Oxytocin and an oxytocin agonist administered centrally decrease food intake in rats. Peptides 12(1), 113-118 (1991)

31. Y. Wu, E. van Dijk, X. Zhou, Evaluating self- vs. other-owned objects: the modulatory role of oxytocin. Biol. Psychol. 92(2), 179-184 (2013). doi:10.1016/j.biopsycho.2012.11.011

32. D. Cai, S. Purkayastha, A new horizon: oxytocin as a novel therapeutic option for obesity and diabetes. Drug Discov. Today Dis. Mech. 10(1-2), e63-e68 (2013). doi:10.1016/j.ddmec.2013.05.006

33. J.E. Blevins, J.M. Ho, Role of oxytocin signaling in the regulation of body weight. Rev. Endocr. Metab. Disord. 14(4), 311-329 (2013). doi:10.1007/s11154-013-9260-x

34. V. Ott, G. Finlayson, H. Lehnert, B. Heitmann, M. Heinrichs, J. Born, M. Hallschmid, Oxytocin reduces reward-driven food intake in humans. Diabetes 62(10), 3418-3425 (2013). doi: $10.2337 / \mathrm{db} 13-0663$

35. W. Qian, T. Zhu, B. Tang, S. Yu, H. Hu, W. Sun, R. Pan, J. Wang, D. Wang, L. Yang, C. Mao, L. Zhou, G. Yuan, Decreased circulating levels of oxytocin in obesity and newly diagnosed type
2 diabetic patients. J. Clin. Endocrinol. Metab. 99(12), 4683-4689 (2014). doi:10.1210/jc.2014-2206

36. N. Cook, J. Miller, J. Hart, Parent observed neuro-behavioral and pro-social improvements with oxytocin following surgical resection of craniopharyngioma. J. Pediatr. Endocrinol. Metab. (2016) doi:10.1515/jpem-2015-0445. (in press)

37. R. Sobota, T. Mihara, A. Forrest, R.E. Featherstone, S.J. Siegel, Oxytocin reduces amygdala activity, increases social interactions, and reduces anxiety-like behavior irrespective of NMDAR antagonism. Behav. Neurosci. 129(4), 389-398 (2015). doi:10.1037/bne0000074

38. K. Lancaster, C.S. Carter, H. Pournajafi-Nazarloo, T. Karaoli, T.S. Lillard, A. Jack, J.M. Davis, J.P. Morris, J.J. Connelly, Plasma oxytocin explains individual differences in neural substrates of social perception. Front. Hum. Neurosci. 9, 132 (2015). doi:10.3389/fnhum.2015.00132

39. M.E. McCullough, P.S. Churchland, A.J. Mendez, Problems with measuring peripheral oxytocin: can the data on oxytocin and human behavior be trusted? Neurosci. Biobehav. Rev 37(8), 1485-1492 (2013). doi:10.1016/j.neubiorev.2013.04.018

40. I.D. Neumann, R. Landgraf, Balance of brain oxytocin and vasopressin: implications for anxiety, depression, and social behaviors. Trends Neurosci. 35(11), 649-659 (2012). doi:10.1016/j.tins.2012.08.004

41. G. Lippi, G.C. Guidi, C. Mattiuzzi, M. Plebani, Preanalytical variability: the dark side of the moon in laboratory testing. Clin. Chem. Lab. Med. 44(4), 358-365 (2006). doi:10.1515/CCLM.2006.073 